\title{
Hole Defects on Two-Dimensional Materials Formed by Electron Beam Irradiation: Toward Nanopore Devices
}

\author{
Hyo Ju Park ${ }^{1,2}$, Gyeong Hee Ryu ${ }^{1,2}$, Zonghoon Lee $e^{1,2, *}$ \\ ${ }^{1}$ School of Materials Science and Engineering, UIsan National Institute of Science and Technology (UNIST), \\ ${ }^{2}$ Center for Multidimensional Carbon Materials, Institute for Basic Science (IBS), Ulsan 44919, Korea
}

*Correspondence to:

Lee $\mathrm{Z}$,

Tel: $+82-52-217-2327$

Fax: +82-52-217-2309

E-mail: zhlee@unist.ac.kr

Received September 4, 2015

Revised September 10, 2015

Accepted September 10, 2015
Two-dimensional (2D) materials containing hole defects are a promising substitute for conventional nanopore membranes like silicon nitride. Hole defects on 2D materials, as atomically thin nanopores, have been used in nanopore devices, such as DNA sensor, gas sensor and purifier at lab-scale. For practical applications of 2D materials to nanopore devices, researches on characteristics of hole defects on graphene, hexagonal boron nitride and molybdenum disulfide have been conducted precisely using transmission electron microscope. Here, we summarized formation, features, structural preference and stability of hole defects on 2D materials with atomic-resolution transmission electron microscope images and theoretical calculations, emphasizing the future challenges in controlling the edge structures and stabilization of hole defects. Exploring the properties at the local structure of hole defects through in situ experiments is also the important issue for the fabrication of realistic $2 \mathrm{D}$ nanopore devices.

Key Words: Two-dimensional materials, Hole defect, Nanopore, Transmission electron microscope, Defect structure

\section{INTRODUCTION}

Defects on two-dimensional (2D) materials were often considered as hindrance despite of potential controllability in electromagnetic and mechanical property of the materials (Lahiri et al., 2010; Yazyev \& Louie, 2010; Attaccalite et al., 2011; Zhou et al., 2013a). For example, the actual performance of graphene devices failed to satisfy the expectations due to the inherent defect in them (Li et al., 2009). Although many efforts to remove the defects in 2D materials (Chen et al., 2013; Lam et al., 2014) have been performed, it has turned out to be only partial healing of the defects. Accordingly, people started to use these inevitable defects to many applications using the fact that defects can manipulate the electrical and magnetic properties of 2D materials. Among various types of defects, the hole defects that are made from ejection of atoms from the sheet were extensively studied for many applications as atomically thin nanopores. In the case of graphene, many studies addressed possibility of hole defects as DNA sequencing (Garaj et al., 2010; Merchant et al., 2010; Schneider et al., 2010; Garaj et al., 2013), gas sensing (Dan et al., 2009), ion and molecules sieve (Sint et al., 2008; Koenig et al., 2012) and many other applications (Liu et al., 2012; $\mathrm{Xu}$ et al., 2013). Several comparable studies also have been done with hexagonal boron nitride (hBN) (Liu et al., 2013a) and molybdenum disulfide $\left(\mathrm{MoS}_{2}\right)$ (Farimani et al., 2014; Liu et al., 2014; Waduge et al., 2015). These studies focus on performance tests at the hole defects without the information of edge configuration and chemical stability which may affect to the performance significantly.

This paper summarizes the ways to produce holes by electron beam irradiation, control the size and shape and stabilize it for the representative $2 \mathrm{D}$ materials including graphene, $\mathrm{hBN}$, and $\mathrm{MoS}_{2}$. Although these three have hexagonal lattices, graphene

This work was supported by Nano Material Technology Development Program through the National Research Foundation of Korea (NRF) funded by the Ministry of Science, ICT and Future Planning (2012M3A7B4049807), and the Korea government (MSIP) (No. 2015R1A2A2A01006992). This work was also supported by IBS-R019-D1.

(a) This is an open-access article distributed under the terms of the Creative Commons Attribution Non-Commercial License (http://creativecommons.org/licenses/by-nc/4.0) which permits unrestricted noncommercial use, distribution, and reproduction in any medium, provided the original work is properly cited.

Copyrights @ 2015 by Korean Society of Microscopy 
consists of mono element of carbon, but hBN contains two elements of boron (B) and nitrogen (N) alternatively in plane, and each molybdenum and sulfur in $\mathrm{MoS}_{2}$ are bound to each other with a ratio of 1:2 in a trigonal prism unit cell wherein Mo layer is sandwiched between sulfur layers (Fig. 1). Each material has its own composition, thus, it shows all different features such as formation process, edge structure, stability and consequential properties of hole defects on graphene, $\mathrm{hBN}$, and $\mathrm{MoS}_{2}$, which are described below.

Among various methods to make holes on 2D materials (Bieri et al., 2009; Girit et al., 2009; Bai et al., 2010; Kim et al., 2010; Koenig et al., 2012; Russo \& Golovchenko, 2012), electron beam in transmission electron microscope (TEM) is good at size control at atomic scale, which is the most important issue for the sensitivity and selectivity of nanopore devices. If electron beam irradiation on a $2 \mathrm{D}$ specimen with a high electron energy breaks the atomic bonds within the material, which is called knock-on voltage, atoms are ejected from the lattice leaving holes on the materials. The atom displacement, knock-on thresholds and other structural information of graphene (Smith \& Luzzi, 2001), hBN (Kotakoski et al., 2010), and $\mathrm{MoS}_{2}$ (Komsa et al., 2012) are summarized in Table 1.

\section{HOLE DEFECTS ON GRPAHENE}

Fig. 2A shows the hole defects of graphene by the electron beam irradiation at $80 \mathrm{kV}$. To make hole defects in graphene, over $86 \mathrm{kV}$ of electron beam energy, the knock-on threshold voltage of graphene, needs to be irradiated on the sample. But some studies showed the existence of oxygen or other chemicals on the sheet or inside TEM chamber can lower the knock-on threshold of graphene by chemical etching effect (Ramasse et al., 2012). Inherent defects created from the synthesizing process of graphene also lower the knockon threshold voltage (Crespi et al., 1996). Once a vacancy is formed, it continuously grows as electron beam irradiates to make an enlarged hole in the sheet. The size of hole defect can be controlled by the time of electron beam irradiation at a given acceleration voltage.

The hole defect of graphene has mixed armchair and zigzag atomic configuration at the edge. Because the edge configuration of graphene are known to have significant influences to the electromagnetic property of graphene (Nakada et al., 1996; Son et al., 2006; Kim \& Kim, 2008; Jung \& MacDonald, 2009; Magda et al., 2014), the edge configuration and its stableness become one of major interests in studies of graphene hole defect. Direct atomic imaging at the graphene
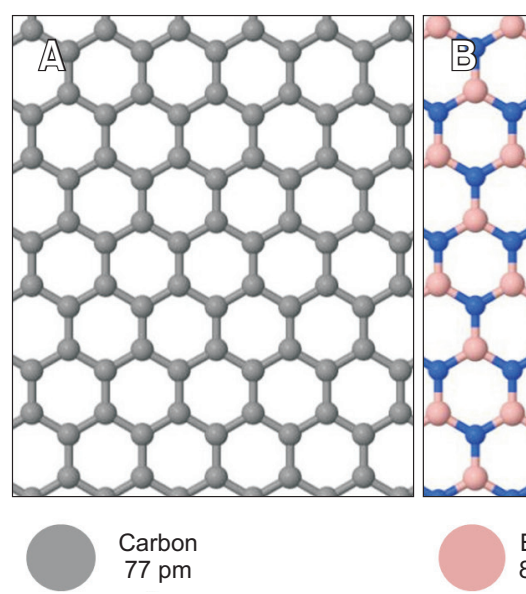
Carbon
$77 \mathrm{pm}$

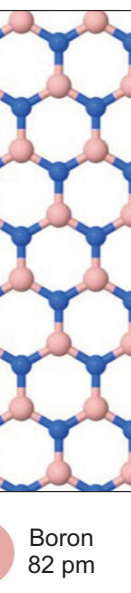

Boron
$82 \mathrm{pm}$

Table 1. Structural data of monolayer graphene, hBN, and $\mathrm{MoS}_{2}(2 \mathrm{H})$

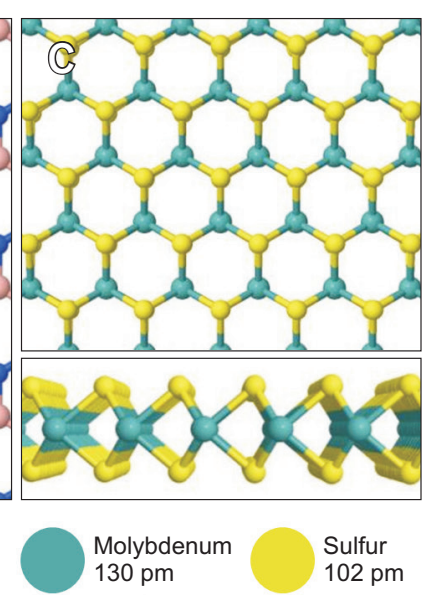

Fig. 1. Atomic models of monolayer graphene, hexagonal boron nitride, and molybdenum disulfide $(2 \mathrm{H})$ from a top and side view with covalent radii of atoms.

\begin{tabular}{|c|c|c|c|c|c|c|}
\hline Reference & Material & Atomic number & Lattice system & Lattice parameter $(\AA)$ & Displacement threshold $(\mathrm{eV})$ & Knock-on threshold (kV) \\
\hline Smith \& Luzzi (2001) & Graphene & C: 6 & Hexagonal & 2.46 & 17 & 86 \\
\hline \multirow[t]{2}{*}{ Kotakoski et al. (2010) } & $\mathrm{hBN}$ & B: 5 & Hexagonal & 2.51 & B: 19 & 79.5 \\
\hline & & $\mathrm{N}: 7$ & & & $\mathrm{~N}: 23$ & 118.6 \\
\hline \multirow[t]{2}{*}{ Komsa et al. (2012) } & $\mathrm{MoS}_{2}(2 \mathrm{H})$ & S: 16 & Hexagonal & 3.2 & S: 6.9 & 80 \\
\hline & & Mo: 42 & & & Mo: 20 & 560 \\
\hline
\end{tabular}

hBN, hexagonal boron nitride; $\mathrm{MoS}_{2}$, molybdenum disulfide.

The calculated values of displacement threshold and knock-on threshold of graphene, hBN, and $\mathrm{MoS}_{2}$ are taken from Smith \& Luzzi (2001) (J. Appl. Phys. 90, 3509-3515); Kotakoski et al. (2010) (Phys. Rev. B 82, 113404); Komsa et al. (2012) (Phys. Rev. Lett. 109, 035503), respectively. 

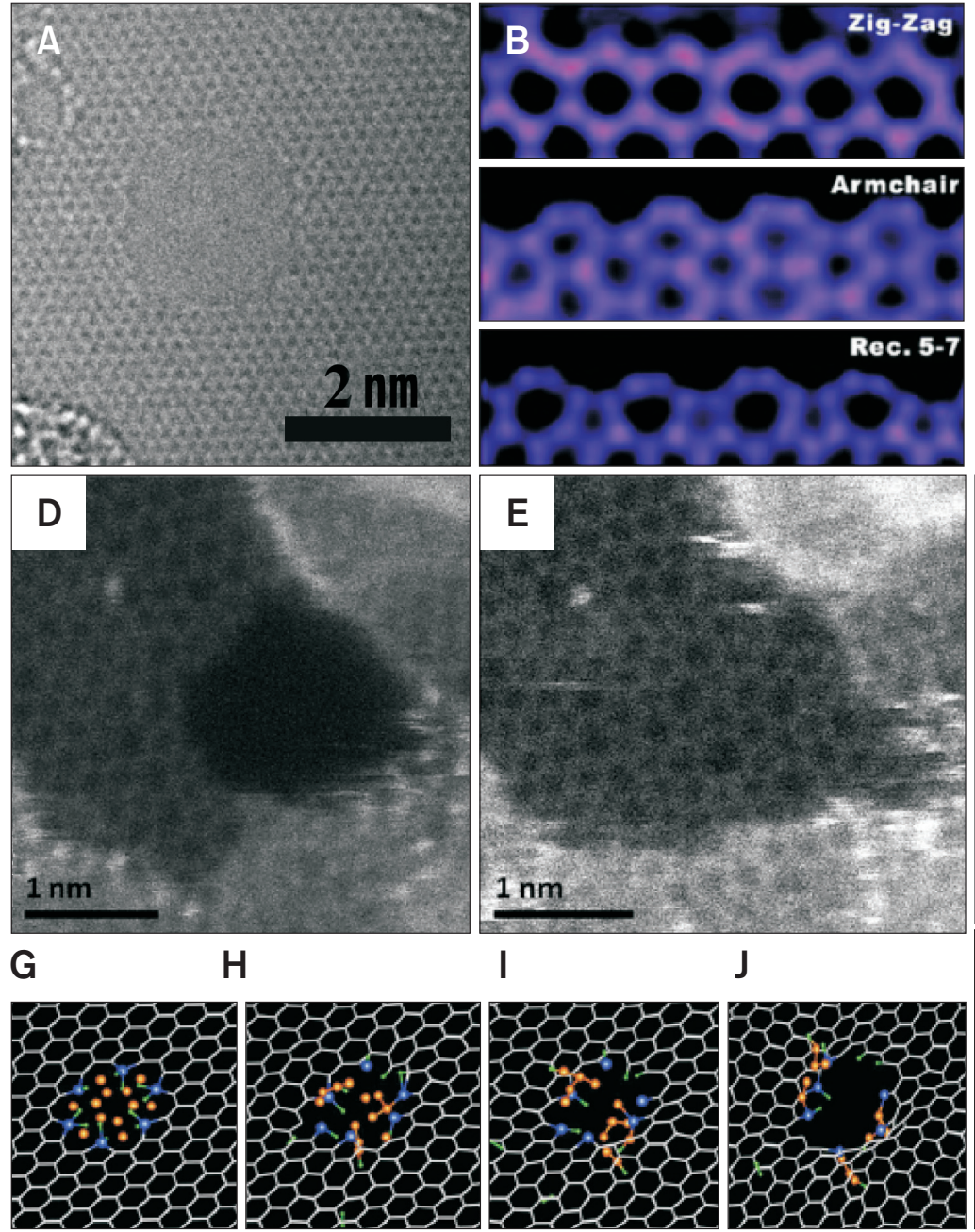

$\mathrm{H}$

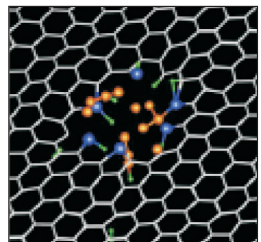

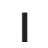

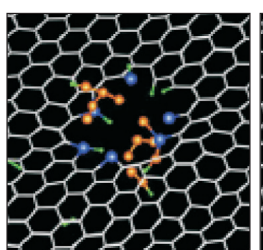

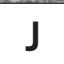

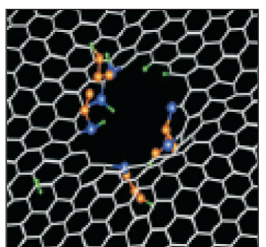

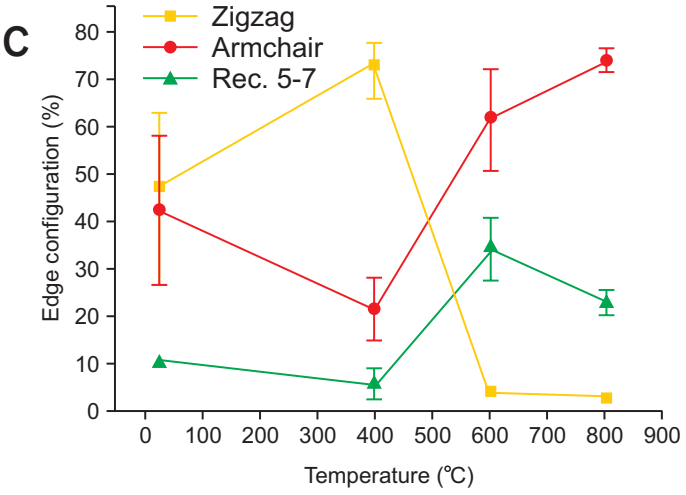
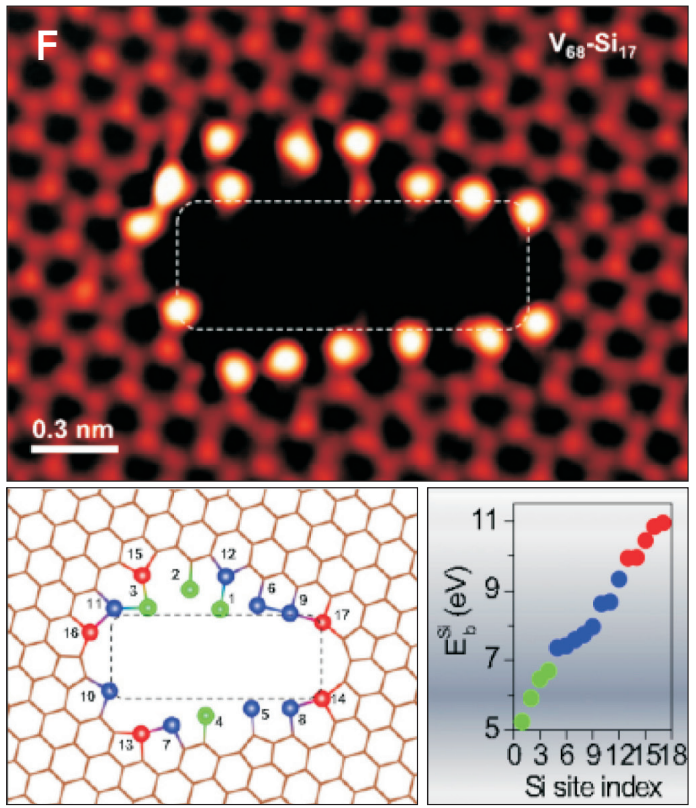

Fig. 2. Features and stability of graphene hole defects. (A) Aberration-corrected transmission electron microscope (TEM) image of graphene hole defect. (B) Graphene has zigzag, armchair, reconstructed (Rec.) 5 to 7 zigzag configuration at the edges. (C) Percentage of each edge configurations is marked on the graph. It shows temperature dependence of edge configurations. (D) Scanning TEM (STEM) image of hole defect created near the hydrocarbon contamination. (E) The hole is filled with C polygons even under ultra high vacuum condition. (F) STEM image and structure model of Si-passivated graphene hole defect and binding energy of Si atoms at the edge. Si-passivated hole defect is stable against hole filling. (G-J) ab initio molecular dynamics simulations reveal the Si-passivated hole forms bonding with $\mathrm{C}$ adatoms out of the graphene plane, preventing the hole filling. Fig. 2B and $\mathrm{C}$ are reprinted from He et al. (2015) (ACS Nano 9, 4786-4795) with original copyright holder's permission. Fig. 2D and E are reprinted from Zan et al. (2012) (Nano Lett. 12, 3936-3940) with original copyright holder's permission. Fig. 2F-J are reprinted from Lee et al. (2014) (Proc. Natl. Acad. Sci. 111, 7522-7526) with original copyright holder's permission.

edges using aberration-corrected TEM gave the insight on the stability of edge configurations (Girit et al., 2009; Song et al., 2011; Kim et al., 2013a; He et al., 2015). Recently, He et al. (2015) reported the temperature dependence of graphene edge configuration through in situ heating experiment using aberration-corrected TEM. As shown in Fig. 2B and $\mathrm{C}$, armchair and reconstructed 5 to 7 zigzag configurations were predominant above $600^{\circ} \mathrm{C}$ which has good agreement with the theoretical predictions (Koskinen et al., 2008). Below $400^{\circ} \mathrm{C}$, zigzag configuration was dominantly observed, which was led by major involvement of chemical etching process from contamination of sample. It gives a prospect in edge control of graphene hole defect for graphene nanopore devices, though contamination effect still remains an issue to be resolved.

Meanwhile, high reactivity of the edges of graphene hole defect has been a big obstacle to realization of graphene nanopore devices. Graphene has high reactivity with chemicals, especially at the hole edges that have dangling bonds. For example, DNA would be fond of sticking to the hole edges and surface when translocating the graphene nanopore, which makes it complicate to identify the DNA sequence (Sathe et al., 2011; Wells et al., 2012). Moreover, despite all the efforts to make holes, small holes in graphene are filled with 
carbon adatoms nearby within hours even under ultra-high vacuum conditions of TEM as shown in Fig. 2D and E (Zan et al., 2012). Thus, fixation or stabilization of hole defects is emerging as a key issue for graphene nanopore devices and passivation of hole edges with other atoms was suggested as one potential method as shown in Fig. 2F-J (Lee et al., 2014). Si-passivated holes in graphene were directly observed using scanning TEM, and proved to be stable against carbon filling even under intense electron beam condition and ambient atmospheric condition. Molecular dynamics simulations supported this observation, showing that carbon adatoms would stick out of the graphene plane preventing filling hole defects. Si passivation opens potential of stabilization of hole defects, however, the fabrication methods should be further developed. Additionally, considering the binding energy between C-Si at armchair site is higher than that at zigzag site, importance of understanding edge configuration of graphene hole defects is highlighted again. Therefore, in-depth studies on the edge configuration and stabilization will make progress toward graphene nanopore devices.

\section{HOLE DEFECTS ON hBN}

Compared to graphene, $\mathrm{hBN}$ has received less attention as a 2D device due to the difficulties in getting synthesized large sheet and non-conducting property (Kim et al., 2013b). As an insulator, $\mathrm{hBN}$ has been used as a substrate of graphene to show high electrical performance (Dean et al., 2010), but not much used alone. However, Liu et al. (2013a) remarked hBN may exhibit superior durability and insulating properties in high-ionic strength solution compared with graphene and realized $\mathrm{hBN}$ nanopore device for DNA sensor.

The notable thing is that hBN hole defects have a unique feature in shape, which is controllable unlike graphene. Fig. $3 \mathrm{~A}-\mathrm{C}$ represent a triangular hole defect in monolayer $\mathrm{hBN}$ sheet. Because boron has a lower knock-on threshold than nitrogen under transmission electron beam (Table 1), $80 \mathrm{kV}$ of electron beam preferentially knock off the boron atoms first, making $\mathrm{B}$ monovacancies with $\mathrm{N}$ terminated edge along the hole defect edge (Jin et al., 2009; Meyer et al., 2009; Kotakoski et al., 2010; Ryu et al., 2015) as shown in Fig. 3A.
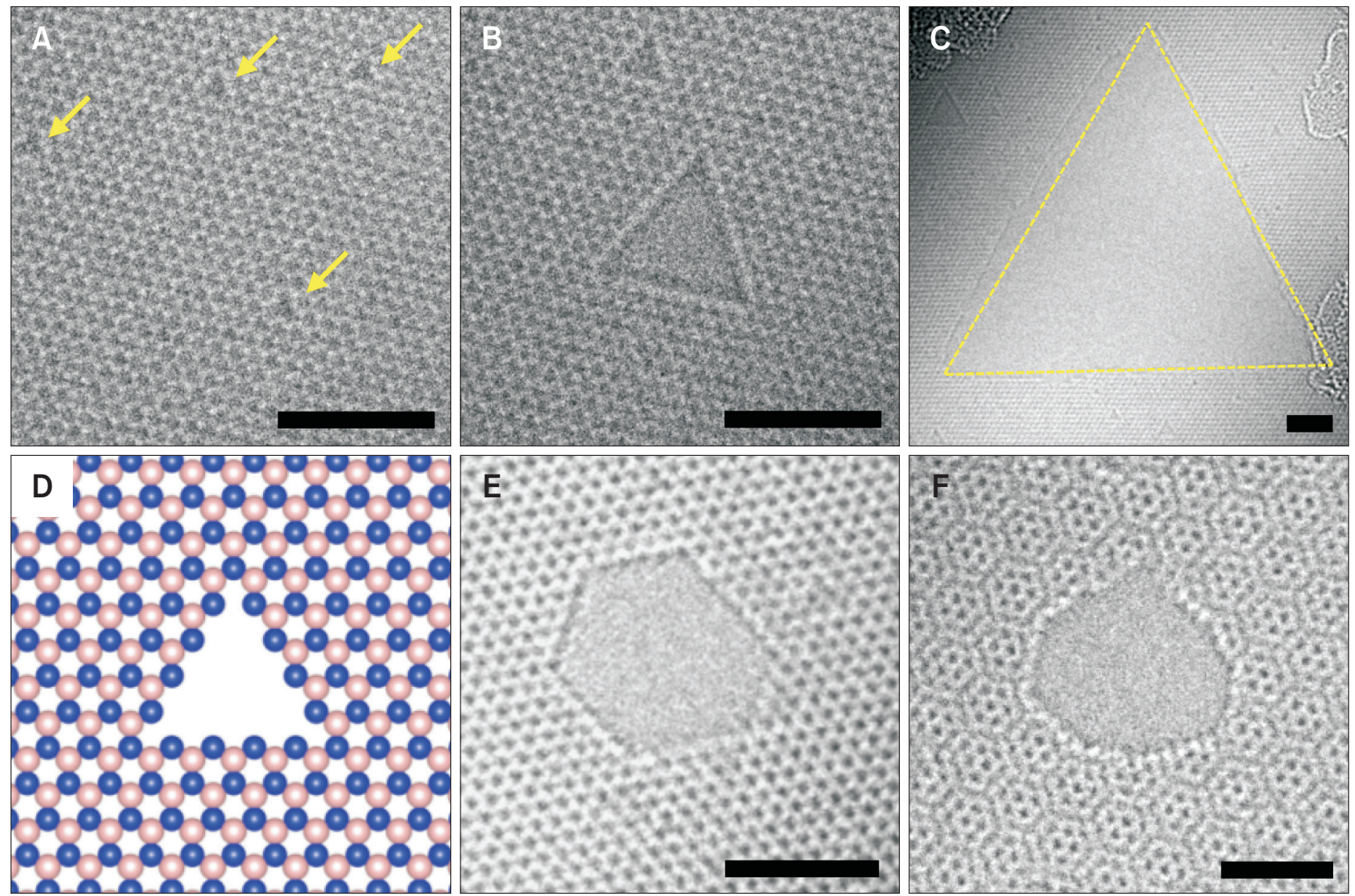

Fig. 3. Hole defects on mono- and double-layer hexagonal boron nitride (hBN). (A-C) In monolayer hBN, hole defects grow with maintaining triangular shape from monovacancy pointed out by yellow arrows (A) to enlarged ( 110 $\left.\mathrm{nm}^{2}\right)$ hole (C) by electron beam irradiation. (D) Atomic model of $\mathrm{N}$-terminated edges of hBN hole defect. The pink and blue balls represent boron and nitrogen atoms, respectively. (E) Hexagonal shape of hole defect is created on AA' stacked hBN double layer. (F) Randomly shaped hole defect is created on rotated hBN double layer. Scale bars=2 nm. 


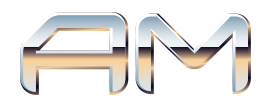

Fig. 3B, the hole defect formed by prolonged electron beam irradiation at Fig. $3 \mathrm{~A}$ is defined as triangular shape more clearly. This triangular hole defect maintains its shape as it grows which is verified up to area of $110 \mathrm{~nm}^{2}$ as shown in Fig. 3C. Atomic model of triangular hole defect in Fig. 3D shows that the edges are terminated with $\mathrm{N}$ atoms. According to the calculation result of Kotakoski et al. (2010), N monovacancy as well as B monovacancy is expected to be formed by above $120 \mathrm{kV}$ of electron beam. Nevertheless, all experimental results using TEM have shown $\mathrm{N}$ terminated triangular hole defect starting from B monovacancy. Yin et al. (2010) explained by cohesive energy calculations that $\mathrm{N}$-terminated hole defect is more stable than B-terminated one due to the ionic character of the material. In our previous work, we found out $\mathrm{N}$-terminated triangular holes are formed even at the edge of the sheet not only inside of the sheet and merging of two holes also results in the restoration of triangular shape (Ryu et al., 2015). Consequently, hole defects created by electron beam on monolayer hBN sheet are always featured by triangular holes with $\mathrm{N}$ terminated edges. Also note that the edges have solely zigzag configuration in case of formation by electron beam, which is another uniqueness compared to the edges of graphene hole defects that have mixed zigzag and armchair configuration.

Furthermore, hexagonal hole defect could be fabricated in AA' stacked double layer hBN sheet like Fig. 3E. The nature of AA' stacking structure of $\mathrm{hBN}$, which $\mathrm{B}$ atoms are on top of $\mathrm{N}$ atoms and vice versa, makes the orientation of defects in one layer 180 degree opposite to those in the other layer of hBN sheet (Meyer et al., 2009). Two opposite triangle defects grow and result in a hexagonal hole defect with $\mathrm{N}$ terminated zigzag edges. Because AA' stacking structure is known to be the most stable structure in bulk hBN structure (Alem et al., 2009), AA' stacked double layer $\mathrm{hBN}$ can be easily obtained through the scotch-tape exfoliation (Pacile et al., 2008). In Fig.
$3 \mathrm{~F}$, if double layer of $\mathrm{hBN}$ is randomly rotated each other by stacking two monolayer of $\mathrm{hBN}$, hole defects have no longer triangular or hexagonal shaped hole defects. The rotated stacking structure can be directly inferred from the Moire pattern. To sum up, ultimately we are able to manufacture freely the hBN nanopores in shape by managing the number of layer and stacking structure, and in size by the irradiation time. Due to these shape and size of hBN hole defects with controllable manners, we are expecting the utilization of the $\mathrm{hBN}$ nanopores as a nano-patterning template. Also $\mathrm{N}$-terminated hole defect of $\mathrm{hBN}$ is found to give enhanced half-metallicity and large magnetism. It suggests the potential of hBN nanopore application for spintronics, light emission and photocatalysis (Du et al., 2009).

\section{HOLE DEFECTS ON MoS}

Recently, $\mathrm{MoS}_{2}$ has been growing interest as a promising material due to its semiconducting nature which makes it facile to make sensing and electronic applications (Farimani et al., 2014; Liu et al., 2014; Waduge et al., 2015). Especially as a DNA detecting sensor, $\mathrm{MoS}_{2}$ nanopore membranes have shown better performance than graphene nanopore membranes for transverse detection without special surface treatment process to prevent the interaction between DNA and the surface, unlike graphene (Wells et al., 2012).

$\mathrm{MoS}_{2}$ is commonly found in $2 \mathrm{H}$ form in nature among three polytypes: $1 \mathrm{~T}, 2 \mathrm{H}$, and 3R. Synthesized $\mathrm{MoS}_{2}$ films may have 3R structures (Wilson \& Yoffe, 1969) but most of studies on $\mathrm{MoS}_{2}$ defects are performed with exfoliated $\mathrm{MoS}_{2}$, so with $2 \mathrm{H}$ structure. The top and side views of $2 \mathrm{H} \mathrm{MoS}_{2}$ are shown in Fig. 1C. The smallest hole in $2 \mathrm{H} \mathrm{MoS}_{2}$ can be created by the removal of one Mo atom or two $S$ atoms but much higher concentration of $S$ site holes than Mo site holes are found in TEM due to the different knock-on threshold of S and Mo
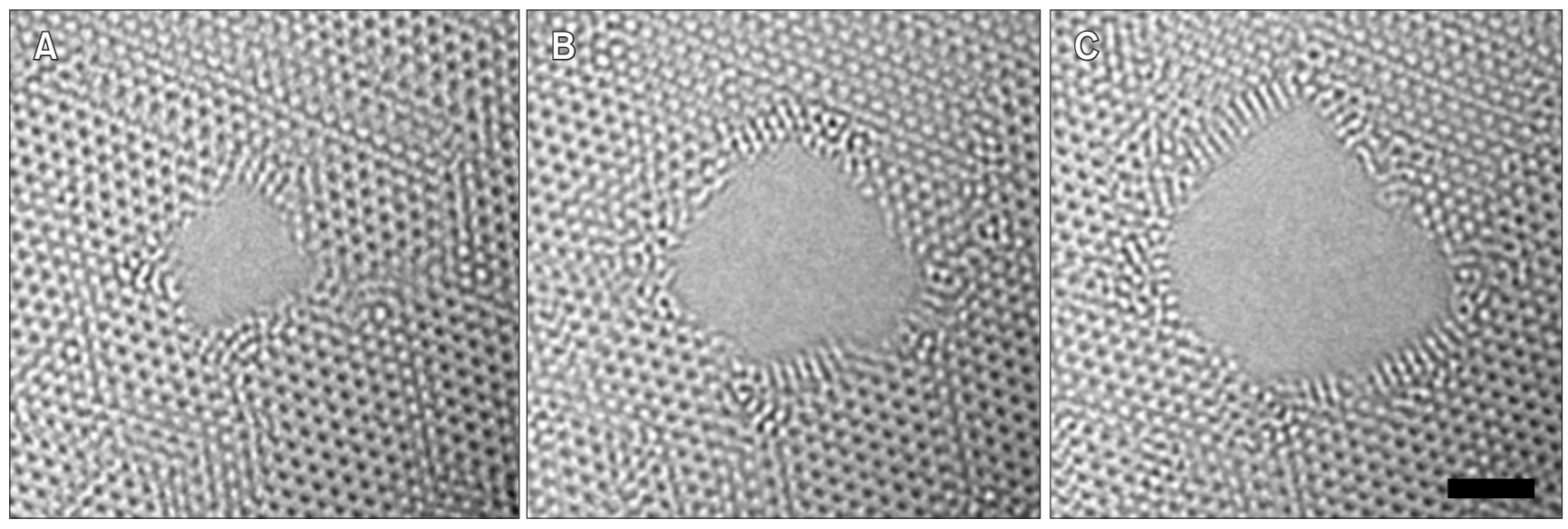

Fig. 4. The sequential growth process of a hole defect on molybdenum disulfide $\left(\mathrm{MoS}_{2}\right)$ by electron beam irradiation in monolayer $\mathrm{MoS}$ sheet. $\mathrm{Mo}$ atoms aggregate at the edge. Scale bar $=2 \mathrm{~nm}$. 
atoms (Hong et al., 2015).

Fig. 4 is the serial images of $\mathrm{MoS}_{2}$ hole defect created by $80 \mathrm{kV}$ of electron beam in TEM. Because the knock-on thresholds of $\mathrm{S}$ and Mo atom are $80 \mathrm{kV}$ and $560 \mathrm{kV}$ respectively as shown in Table 1, S vacancies are formed first by slightly focused electron beams. Meanwhile Mo atoms are less likely to be ejected by $80 \mathrm{kV}$ electron beam, resulting in the agglomeration of Mo atoms at the edge of hole defects. From Fig. 4, Mo atoms increasingly aggregate at the edge under continued electron beam irradiation. Although a few papers reported the phenomenon of Mo atoms agglomeration (Liu et al., 2013b; Zan et al., 2013), none reported the corresponding effects in electrical or magnetic properties at the edge. According to the theoretical calculations (Zhou et al., 2013b), Mo-Mo metallic bonds are formed at $\mathrm{S}$ vacancy sites and cancel the magnetism by pairing the unsaturated spin electrons. Similarly, the degree of Mo atoms agglomeration may affect to the metallicity so to the electrical performance such as the current signals when DNA or molecules transverse the holes. Therefore, for $\mathrm{MoS}_{2}-$ based nanopore devices, understanding of edge configuration of $\mathrm{MoS}_{2}$ hole defects and consequent electrical and magnetic properties is required.

\section{CONCLUSIONS}

Hole defects on 2D materials have been studied extensively from fabrication, features, control to stabilization. In summary, graphene hole defects have mixed armchair, zigzag and reconstructed 5 to 7 zigzag configurations at the edge which are temperature-dependent. Due to the high reactivity of graphene and its hole defect with chemicals, additional process of stabilization is required. $\mathrm{hBN}$ hole defects have advantage in shape control to triangle, hexagon and randomly shaped hole by managing the number of layers and the stacking structure. And it always has N-terminated zigzag edges which make it possible to control the electromagnetic properties for nanopore devices. $\mathrm{MoS}_{2}$ hole defects show Mo atoms agglomeration at the edges. Based on these studies, 2D materials nanopores become more realizable. TEM experiments have made a significant contribution to understanding of hole defects on 2D materials by direct imaging of the features and dynamics of hole defects; however, interconnecting the structure and property of hole defects was lack. In situ experiments using the real-time imaging and performance measurement in TEM would accelerate the realization of $2 \mathrm{D}$ materials-based nanopore devices.

\section{EXPERIMENTAL METHODS}

Some images except reprinted ones with permission from references in figures were taken by the authors of this paper using an aberration-corrected FEI Titan Cube TEM (FEI $\operatorname{Titan}^{3}$ G2 60-300; FEI, Netherlands) with a monochromator at $80 \mathrm{kV}$. Imaging condition was settled as $-21 \pm 0.5 \mu \mathrm{m}$ of spherical aberration (Cs), around $5 \times 10^{5} \mathrm{e}^{-} \mathrm{nm}^{-2}$ of electron beam densities. Images were taken with 0.5 seconds of exposure time.

\section{CONFLICT OF INTEREST}

No potential conflict of interest relevant to this article was reported.

\section{REFERENCES}

Alem N, Erni R, Kisielowski C, Rossell M D, Gannett W, and Zettl A (2009) Atomically thin hexagonal boron nitride probed by ultrahigh-resolution transmission electron microscopy. Phys. Rev. B 80, 155425.

Attaccalite C, Bockstedte M, Marini A, Rubio A, and Wirtz L (2011) Coupling of excitons and defect states in boron-nitride nanostructures. Phys. Rev. B 83, 144115.

Bai J, Zhong X, Jiang S, Huang Y, and Duan X (2010) Graphene nanomesh. Nat. Nanotechnol. 5, 190-194.

Bieri M, Treier M, Cai J, Ait-Mansour K, Ruffieux P, Groning O, Groning P, Kastler M, Rieger R, Feng X, Mullen K, and Fasel R (2009) Porous graphenes: two-dimensional polymer synthesis with atomic precision. Chem. Commun. (45), 6919-6921.

Chen J H, Shi T W, Cai T C, Xu T, Sun L T, Wu X S, and Yu D P (2013) Self healing of defected graphene. Appl. Phys. Lett. 102, 103107.

Crespi V H, Chopra N G, Cohen M L, Zettl A, and Louie S G (1996) Anisotropic electron-beam damage and the collapse of carbon nanotubes. Phys. Rev. B 54, 5927-5931.

Dan Y, Lu Y, Kybert N J, Luo Z, and Johnson A T (2009) Intrinsic response of graphene vapor sensors. Nano Lett. 9, 1472-1475.

Dean C R, Young A F, Meric I, Lee C, Wang L, Sorgenfrei S, Watanabe K, Taniguchi T, Kim P, Shepard K L, and Hone J (2010) Boron nitride substrates for high-quality graphene electronics. Nat. Nanotechnol. 5, 722-726.

Du A, Chen Y, Zhu Z, Amal R, Lu G Q, and Smith S C (2009) Dots versus antidots: computational exploration of structure, magnetism, and half-metallicity in boron-nitride nanostructures. J. Am. Chem. Soc. 131, 17354-17359.

Farimani A B, Min K, and Aluru N R (2014) DNA base detection using a single-layer MoS2. Acs Nano 8, 7914-7922.

Garaj S, Hubbard W, Reina A, Kong J, Branton D, and Golovchenko J A (2010) Graphene as a subnanometre trans-electrode membrane. Nature 467, 190-193.

Garaj S, Liu S, Golovchenko J A, and Branton D (2013) Molecule-hugging graphene nanopores. Proc. Natl. Acad. Sci. 110, 12192-12196.

Girit C O, Meyer J C, Erni R, Rossell M D, Kisielowski C, Yang L, Park C H, Crommie M F, Cohen M L, Louie S G, and Zettl A (2009) Graphene at 
the edge: stability and dynamics. Science $\mathbf{3 2 3}, \mathbf{1 7 0 5 - 1 7 0 8 .}$

He K, Robertson A W, Fan Y, Allen C S, Lin Y C, Suenaga K, Kirkland A I, and Warner J H (2015) Temperature dependence of the reconstruction of zigzag edges in graphene. ACS Nano 9, 4786-4795.

Hong J, Hu Z, Probert M, Li K, Lv D, Yang X, Gu L, Mao N, Feng Q, Xie L, Zhang J, Wu D, Zhang Z, Jin C, Ji W, Zhang X, and Yuan J (2015) Exploring atomic defects in molybdenum disulphide monolayers. Nat. Commun. 6, 6293.

Jin C, Lin F, Suenaga K, and lijima S (2009) Fabrication of a freestanding boron nitride single layer and its defect assignments. Phys. Rev. Lett. 102, 195505.

Jung J and MacDonald A H (2009) Carrier density and magnetismin graphene zigzag nanoribbons. Phys. Rev. B 79, 235433.

Kim G, Jang A R, Jeong H Y, Lee Z, Kang D J, and Shin H S (2013b) Growth of high-crystalline, single-layer hexagonal boron nitride on recyclable platinum foil. Nano Lett. 13, 1834-1839.

Kim K, Coh S, Kisielowski C, Crommie M F, Louie S G, Cohen M L, and Zettl A (2013a) Atomically perfect torn graphene edges and their reversible reconstruction. Nat. Commun. 4, 2723.

Kim M, Safron N S, Han E, Arnold M S, and Gopalan P (2010) Fabrication and characterization of large-area, semiconducting nanoperforated graphene materials. Nano Lett. 10, 1125-1131.

Kim W Y and Kim K S (2008) Prediction of very large values of magnetoresistance in a graphene nanoribbon device. Nat. Nanotechnol. 3, 408-412.

Koenig S P, Wang L, Pellegrino J, and Bunch J S (2012) Selective molecular sieving through porous graphene. Nat. Nanotechnol. 7, 728-732.

Komsa H P, Kotakoski J, Kurasch S, Lehtinen O, Kaiser U, and Krasheninnikov A V (2012) Two-dimensional transition metal dichalcogenides under electron irradiation: defect production and doping. Phys. Rev. Lett. 109, 035503.

Koskinen P, Malola S, and Hakkinen H (2008) Self-passivating edge reconstructions of graphene. Phys. Rev. Lett. 101, 115502.

Kotakoski J, Jin C H, Lehtinen O, Suenaga K, and Krasheninnikov A $\checkmark$ (2010) Electron knock-on damage in hexagonal boron nitride monolayers. Phys. Rev. B 82, 113404.

Lahiri J, Lin Y, Bozkurt P, Oleynik I I, and Batzill M (2010) An extended defect in graphene as a metallic wire. Nat. Nanotechnol. 5, 326-329.

Lam D V, Kim S M, Cho Y, Kim J H, Lee H J, Yang J M, and Lee S M (2014) Healing defective CVD-graphene through vapor phase treatment. Nanoscale 6, 5639-5644.

Lee J, Yang Z, Zhou W, Pennycook S J, Pantelides S T, and Chisholm M F (2014) Stabilization of graphene nanopore. Proc. Natl. Acad. Sci. 111, 7522-7526.

Li X S, Cai W W, An J H, Kim S, Nah J, Yang D X, Piner R, Velamakanni A, Jung I, Tutuc E, Banerjee S K, Colombo L, and Ruoff R S (2009) Large-area synthesis of high-quality and uniform graphene films on copper foils. Science 324, 1312-1314.

Liu K, Feng J, Kis A, and Radenovic A (2014) Atomically thin molybdenum disulfide nanopores with high sensitivity for DNA translocation. Acs Nano 8, 2504-2511.

Liu S, Lu B, Zhao Q, Li J, Gao T, Chen Y, Zhang Y, Liu Z, Fan Z, Yang F, You L, and Yu D (2013a) Boron nitride nanopores: highly sensitive DNA single-molecule detectors. Adv. Mater. 25, 4549-4554.

Liu S, Zhao Q, Xu J, Yan K, Peng H, Yang F, You L, and Yu D (2012) Fast and controllable fabrication of suspended graphene nanopore devices. Nanotechnology 23, 085301.

Liu X, Xu T, Wu X, Zhang Z, Yu J, Qiu H, Hong J H, Jin C H, Li J X, Wang X R, Sun L T, and Guo W (2013b) Top-down fabrication of sub-nanometre semiconducting nanoribbons derived from molybdenum disulfide sheets. Nat. Commun. 4, 1776.

Magda G Z, Jin X, Hagymasi I, Vancso P, Osvath Z, Nemes-Incze P, Hwang C, Biro L P, and Tapaszto L (2014) Room-temperature magnetic order on zigzag edges of narrow graphene nanoribbons. Nature $\mathbf{5 1 4}$, 608611.

Merchant C A, Healy K, Wanunu M, Ray V, Peterman N, Bartel J, Fischbein M D, Venta K, Luo Z, Johnson A T, and Drndic M (2010) DNA translocation through graphene nanopores. Nano Lett. 10, 29152921.

Meyer J C, Chuvilin A, Algara-Siller G, Biskupek J, and Kaiser U (2009) Selective sputtering and atomic resolution imaging of atomically thin boron nitride membranes. Nano Lett. 9, 2683-2689.

Nakada K, Fujita M, Dresselhaus G, and Dresselhaus M S (1996) Edge state in graphene ribbons: nanometer size effect and edge shape dependence. Phys. Rev. B 54, 17954-17961.

Pacile D, Meyer J C, Girit C O, and Zettl A (2008) The two-dimensional phase of boron nitride: few-atomic-layer sheets and suspended membranes. Appl. Phys. Lett. 92, 133107.

Ramasse Q M, Zan R, Bangert U, Boukhvalov D W, Son Y W, and Novoselov K S (2012) Direct experimental evidence of metalmediated etching of suspended graphene. ACS Nano 6, 4063-4071.

Russo C J and Golovchenko J A (2012) Atom-by-atom nucleation and growth of graphene nanopores. Proc. Natl. Acad. Sci. 109, 59535957.

Ryu G H, Park H J, Ryou J, Park J, Lee J, Kim G, Shin H S, Bielawski C W, Ruoff R S, Hong S, and Lee Z (2015) Atomic-scale dynamics of triangular hole growth in monolayer hexagonal boron nitride under electron irradiation. Nanoscale 7, 10600-10605.

Sathe C, Zou X, Leburton J P, and Schulten K (2011) Computational investigation of DNA detection using graphene nanopores. ACS Nano 5, 8842-8851.

Schneider G F, Kowalczyk S W, Calado V E, Pandraud G, Zandbergen H W, Vandersypen L M, and Dekker C (2010) DNA translocation through graphene nanopores. Nano Lett. 10, 3163-3167.

Sint K, Wang B, and Kral P (2008) Selective ion passage through functionalized graphene nanopores. J. Am. Chem. Soc. 130, 1644816449.

Smith B W and Luzzi D E (2001) Electron irradiation effects in single wall carbon nanotubes. J. Appl. Phys. 90, 3509-3515.

Son Y W, Cohen M L, and Louie S G (2006) Half-metallic graphene nanoribbons. Nature 444, 347-349.

Song B, Schneider G F, Xu Q, Pandraud G, Dekker C, and Zandbergen H (2011) Atomic-scale electron-beam sculpting of near-defect-free graphene nanostructures. Nano Lett. 11, 2247-2250.

Waduge P, Bilgin I, Larkin J, Henley R Y, Goodfellow K, Graham A C, Bell D C, Vamivakas N, Kar S, and Wanunu M (2015) Direct and scalable deposition of atomically thin low-noise MoS2 membranes on apertures. Acs Nano 9, 7352-7359.

Wells D B, Belkin M, Comer J, and Aksimentiev A (2012) Assessing graphene nanopores for sequencing DNA. Nano Lett. 12, 41174123.

Wilson J A and Yoffe A D (1969) Transition metal dichalcogenides discussion and interpretation of observed optical, electrical and 
structural properties. Adv. Phys. 18, 193-335.

Xu Q, Wu M Y, Schneider G F, Houben L, Malladi S K, Dekker C, Yucelen E, Dunin-Borkowski R E, and Zandbergen H W (2013) Controllable atomic scale patterning of freestanding monolayer graphene at elevated temperature. Acs Nano 7, 1566-1572.

Yazyev O V and Louie S G (2010) Electronic transport in polycrystalline graphene. Nat. Mater. 9, 806-809.

Yin L C, Cheng H M, and Saito R (2010) Triangle defect states of hexagonal boron nitride atomic layer: density functional theory calculations. Phys. Rev. B 81, 153407.

Zan R, Ramasse Q M, Bangert U, and Novoselov K S (2012) Graphene reknits its holes. Nano Lett. 12, 3936-3940.

Zan R, Ramasse Q M, Jalil R, Georgiou T, Bangert U, and Novoselov K S (2013) Control of radiation damage in MoS2 by graphene encapsulation. ACS Nano 7, 10167-10174.

Zhou W, Zou X L, Najmaei S, Liu Z, Shi Y M, Kong J, Lou J, Ajayan P M, Yakobson B I, and Idrobo J C (2013a) Intrinsic structural defects in monolayer molybdenum disulfide. Nano Lett. 13, 2615-2622.

Zhou Y, Yang P, Zu H, Gao F, and Zu X (2013b) Electronic structures and magnetic properties of MoS2 nanostructures: atomic defects, nanoholes, nanodots and antidots. Phys. Chem. Chem. Phys. 15, 10385-10394. 